\title{
Trainer für die Hoffnung
}

Erhard Taverna kung durch zwei Berater aus der Schweiz, von Wilhelm Felder, Jahrgang 1947, bis 2012 Ordinarius für Kinder- und Jugendpsychiatrie an der Universi-

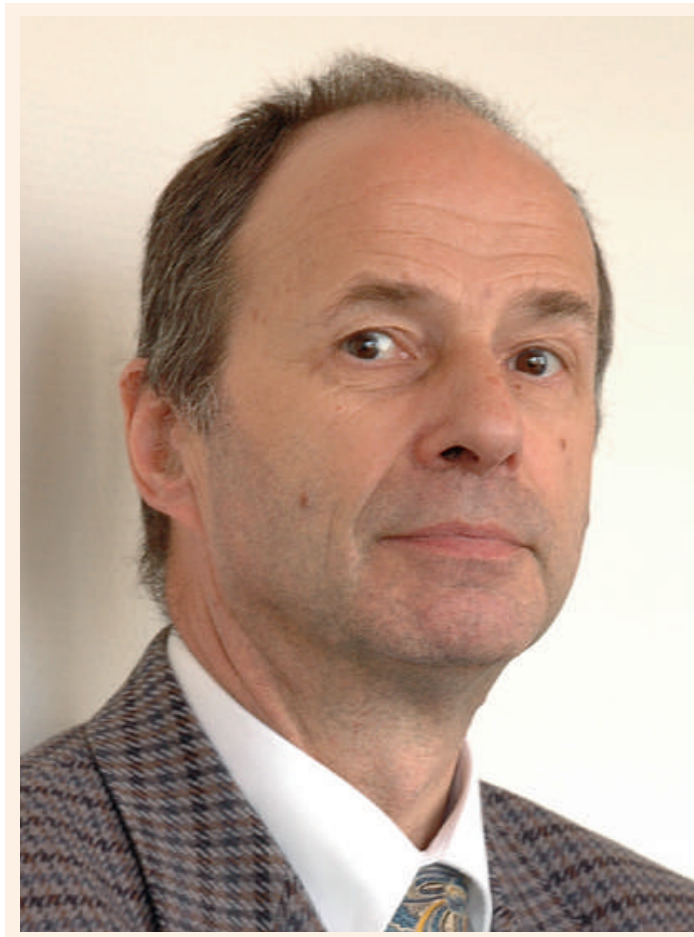

Verstärken das Team von Ruedy Lüthy in Harare: Wilhelm Felder ... tät Bern und Direktor der gleichnamigen Klinik, sowie von Ruedi Zollinger, Jahrgang 1947, bis 2012 Chefarzt Kinder- und Jugendpsychiatrische Dienste St. Gallen. Inzwischen haben beide drei Mal zusammen die Klinik in Harare besucht und sich mit der Situation der Behandelnden und deren Patienten vertraut gemacht. Als wichtigstes Ziel gehe es darum die Therapie und damit die Überlebenschancen der Kinder und Jugendlichen zu verbessern, die aus ihrer Depression heraus die Behandlung vernachlässigten. Die Medikamente müssen alle 12 Stunden eingenommen und dürfen innerhalb von drei Wochen

\section{Als wichtigstes Ziel gehe es darum, die Therapie [...] der Kinder und Jugendlichen zu verbessern, die aus ihrer Depression heraus die Behandlung vernachlässigten.}

nur einmal vergessen werden, weil sonst Gefahr von Resistenzen besteht. Regelmässig kommen die 15- bis 25-Jährigen in die Tagesklinik, wo sie medizinische Hilfe, Anleitung zur Selbstversorgung und auch Nah-

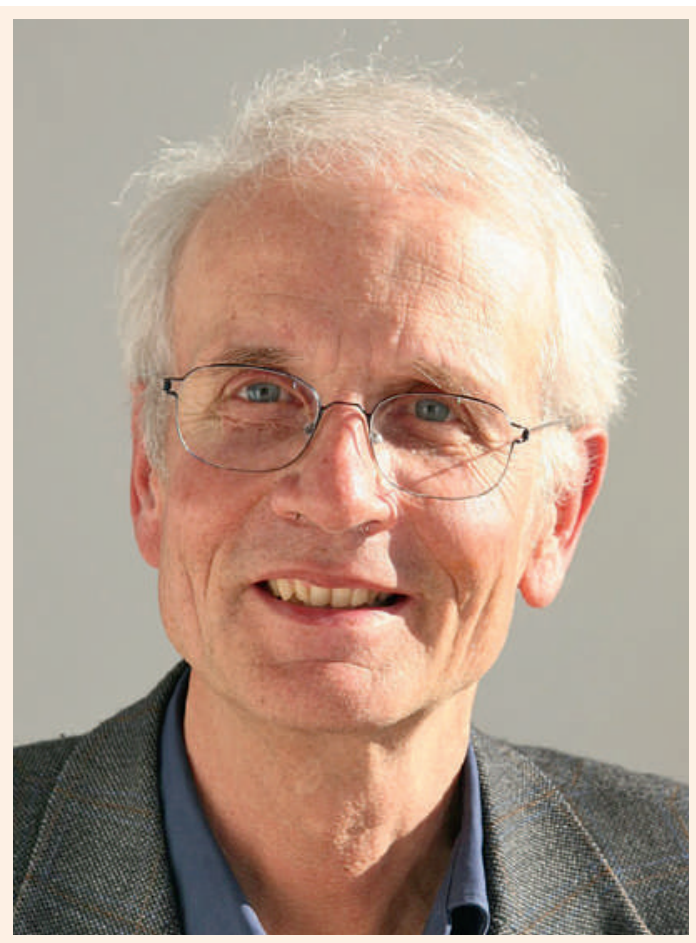

... und Ruedi Zollinger. 
rungsmittelhilfe bekommen und sich Wissen über die HIV-Prävention aneignen. Nur jedes zweite HIVpositive Baby, das bei der Geburt oder beim Stillen angesteckt wurde, überlebt. Viele dieser Überlebenden sind kleinwüchsig und emotional wie intellektuell retardiert. Das Projekt, das Wilhelm Felder und Ruedi Zollinger ausgearbeitet haben, ist ambitioniert und, je nach finanzieller Unterstützung, auf vier bis fünf Jahre ausgelegt. Vorgesehen sind vier Teilprojekte: A. Empowerment des Klinikpersonals im psychosozialen Bereich, B. Assessment von Jugendlichen im psychosozialen Bereich, C. Gruppentherapeutische Angebote und D. Tagesstrukturen für Kinder und Jugendliche. tragen. Konkurrenz erhält die verbliebene universitäre Medizin vor allem durch christliche Sekten, die Prävention und Therapie ablehnen oder durch traditionelle Heiler, die ihre magischen Methoden vorziehen.

Die beiden Consultants haben sich für ihre Pensionierungsjahre eine schwierige Aufgabe ausgesucht. Vieles entspreche der gewohnten Klinikerfahrung unter erschwerten Bedingungen, ausserdem würden sie eine Menge über die HIV-Infektionen und das heutige Zimbabwe lernen. Ausser einer Spesenentschädigung bekommen sie keinen Lohn. Die Newlands Clinic wird von der Stiftung Swiss Aids Care International mit Sitz in Zürich finanziert und

\section{Wer eine Resistenz entwickelt, bekommt eine zweite Chance, nicht aber, wie in der Schweiz, eine dritte und vierte.}

Beide Berater hatten es mit ähnlichen fachlichen Problemen, wie in der Schweiz, zu tun. Was zur fehlenden Akzeptanz der chronischen Krankheit und der Stigmatisierung dazukomme, seien die extreme Armut, das schwierige Umfeld der Slums und die meist fehlende Unterstützung durch Angehörige. Zimbabwe ist in einem sehr schlechten Zustand, Wirtschaft und Bildungswesen darben, ausgebildete Fachkräfte und junge Menschen verlassen das Land. Die Ausbildung des gut motivierten, einheimischen Personals richtet sich vor allem an Pfleger und Pflegerinnen, die im Gesundheitswesen die Hauptlast

\section{Interaktiver Artikel}

Wollen Sie diesen Artikel kommentieren? Nutzen Sie dafür die Kommentarfunktion in der OnlineVersion oder sehen Sie nach, was Ihre Kolleginnen und Kollegen bereits geschrieben haben: www.saez.ch/aktuelle-ausgabe/interaktive-beitraege/ betrieben. Die gratis abgegeben Medikamente erhält sie in erster Linie vom Global Fund und der ClintonHealth-Access-Initiative. Ausserdem unterstützen die DEZA sowie zahlreiche institutionelle und private Spender die Arbeit. Ein eigenes Labor bestimmt neben der Routine die im Blut zirkulierenden HIViren und Immunzellen. Blutspiegelbestimmungen von Medikamenten oder Resistenzprüfungen sind aus Kostengründen nicht möglich. Wer eine Resistenz entwickelt, bekommt eine zweite Chance, nicht aber, wie in der Schweiz, eine dritte und vierte mit sehr viel teureren Medikamenten. Auch aus diesem Grund komme der Motivation zur regelmässigen Medikamenteneinnahme eine grosse Bedeutung zu. Sowohl Willi Felder wie auch Ruedi Zollinger sind gerne bereit, vor interessierten Sponsoren oder bei medizinischen Fortbildungen über ihre Arbeit in Harare zu berichten.

- www.swissaidscare.ch - www.facebook.com/ swissaidscare

- www.newlandsclinic.org.zw - wilhelm.felder[at]hin.ch ruedi.zollinger[at]bluewin.ch 